ANNALES

POLONICI MATHEMATICI

LVII.3 (1992)

\title{
Differential conditions to verify the Jacobian Conjecture
}

\author{
by Ludwik M. DrużKowski and Halszka K. TutaJ (Kraków)
}

Abstract. Let $F$ be a polynomial mapping of $\mathbb{R}^{2}, F(0)=0$. In 1987 Meisters and Olech proved that the solution $y(\cdot)=0$ of the autonomous system of differential equations $\dot{y}=F(y)$ is globally asymptotically stable provided that the jacobian of $F$ is everywhere positive and the trace of the matrix of the differential of $F$ is everywhere negative. In particular, the mapping $F$ is then injective. We give an $n$-dimensional generalization of this result.

1. Introduction. The following problem was explicitly stated by Markus and Yamabe (cf. [MY], [O]).

Global Stability Problem in $\mathbb{R}^{2}$. Let $F=\left(F_{1}, F_{2}\right)$ be a $\mathcal{C}^{1}$ transformation of $\mathbb{R}^{2}, F(0,0)=(0,0)$. Assume that the matrix of the differential of $F$ has, at any point $x$ of $\mathbb{R}^{2}$, all eigenvalues with negative real parts; that is, assume that

$$
\operatorname{Jac} F\left(x_{1}, x_{2}\right)>0 \quad \text { for every }\left(x_{1}, x_{2}\right) \in \mathbb{R}^{2}
$$

and

$$
\begin{aligned}
& \operatorname{Tr} F^{\prime}\left(x_{1}, x_{2}\right):=\frac{\partial F_{1}}{\partial x_{1}}\left(x_{1}, x_{2}\right)+\frac{\partial F_{2}}{\partial x_{2}}\left(x_{1}, x_{2}\right)<0 \\
& \quad \text { for every }\left(x_{1}, x_{2}\right) \in \mathbb{R}^{2} .
\end{aligned}
$$

Does it then follow that the solution $\left(x_{1}, x_{2}\right)=(0,0)$ of the autonomous system of differential equations

$$
\dot{x}_{1}(t)=F_{1}\left(x_{1}, x_{2}\right), \quad \dot{x}_{2}(t)=F_{2}\left(x_{1}, x_{2}\right)
$$

is globally asymptotically stable? That is, does every solution curve of (*) approach $(0,0)$ as $t \rightarrow \infty$ ?

1991 Mathematics Subject Classification: 26B10, 34D99,26C10.

Key words and phrases: jacobian conditions, global injectivity, global stability. 
It has been showed that the problem has an affirmative solution under some additional conditions (cf. e.g. $[\mathrm{H}],[\mathrm{HO}],[\mathrm{MO}],[\mathrm{MY}],[\mathrm{O}],[\mathrm{P}]$ ). In particular, Meisters and Olech proved in $[\mathrm{MO}]$ that the answer is positive provided $F$ is a polynomial mapping. As a consequence they showed that a polynomial mapping $F$ is injective, which was a partial affirmative answer to the Global Univalence Problem formulated by Olech (cf. [O]):

Global Univalence Problem in $\mathbb{R}^{2}$. Is a $\mathcal{C}^{1}$ mapping $F$ of $\mathbb{R}^{2}$ globally univalent (i.e. injective) provided that the assumptions $(\mathrm{J})$ and $(\mathrm{T})$ are satisfied?

Note that the Global Univalence Problem is equivalent to the Global Stability Problem (cf. [O]) and it is still not settled. To show how delicate the matter is we recall the following example presented in $[\mathrm{P}]$.

EXAMPLE 1.1. Define an analytic map $F$ of $\mathbb{R}^{2}$ by

$$
F(x, y)=\left(-2 e^{x}+3 y^{2}-1, y e^{x}-y^{3}\right) .
$$

Then $\operatorname{Jac} F(x, y)=-2 e^{x}<0$ and $\operatorname{Tr} F^{\prime}(x, y)=-e^{x}-3 y^{2}<0$ for every $(x, y) \in \mathbb{R}^{2}$, but $F$ is not injective because $F(0,1)=(0,0)=F(0,-1)$.

The Global Stability Problem in $\mathbb{R}^{2}$ has a natural formulation in any dimension:

Global Stability Problem in $\mathbb{R}^{n}$. Let $F=\left(F_{1}, \ldots, F_{n}\right)$ be a $\mathcal{C}^{1}$ transformation of $\mathbb{R}^{n}, F(0)=0$ and assume that for any $x$ in $\mathbb{R}^{n}$ all eigenvalues of the jacobian matrix $F^{\prime}(x)$ have negative real parts. Does it then follow that the solution $y(\cdot)=0$ of the autonomous system of differential equations

$$
\dot{y}=F(y)
$$

is globally asymptotically stable?

The negative answer to the Global Stability Problem in $\mathbb{R}^{n}$ for $n \geq 4$ was given in 1988 by N. E. Barabanov (cf. [B]); the problem is still open when $n=2,3$.

In this paper we give an $n$-dimensional generalization of Meisters and Olech's just mentioned two-dimensional result (see Section 4 for the precise lormulation).

2. Basic facts on stability. Let $E$ be a subset of $\mathbb{R}^{n}$ and $F: E \rightarrow \mathbb{R}^{n}$ be a $\mathcal{C}^{1}$ mapping. Consider a real autonomous system of differential equations

$$
\dot{y}=F(y)
$$

whose solutions are uniquely determined by initial conditions. Let $y_{0}(\cdot)$ denote the solution of $(*)$ satisfying the initial condition $y_{0}(0)=y_{0}$ and defined 
for every $t \geq 0$. In the sequel we shall assume that $F(0)=0$ and $y_{0}=0$, so $y_{0}(\cdot)=0$.

The symbol of matrix multiplication is omitted or denoted by "o", "T" is the matrix tranposition and $x$ is treated as one-column matrix, so $x^{T}$ is a one-row matrix. The norm $\|\cdot\|$ is the euclidean norm in $\mathbb{R}^{n}$, and $I$ denotes the identity mapping or the identity matrix.

We start with a series of definitions.

DEFINITION 2.1. (i) We say that $y_{0}(\cdot)$ is locally asymptotically stable (for short: LAS) if for every $\varepsilon>0$ there exists $\delta=\delta_{\varepsilon}>0$ such that if $\left\|y_{0}-y_{1}\right\|<\delta$ then the solution $y_{1}(\cdot)$ of $(*)$ with $y_{1}(0)=y_{1}$ exists for every $t \geq 0,\left\|y_{0}(t)-y_{1}(t)\right\|<\varepsilon$ for $t \geq 0$ and $\left\|y_{0}(t)-y_{1}(t)\right\| \rightarrow 0$ as $t \rightarrow \infty$.

(ii) The solution $y_{0}(\cdot)$ is globally asymptotically stable (for short: GAS) when it is a LAS solution and the following holds:

If $y_{1}(\cdot)$ is any solution of $(*)$ defined for small $t \geq 0$, then $y_{1}(t)$ exists for all $t \geq 0$ and $\left\|y_{0}(t)-y_{1}(t)\right\| \rightarrow 0$ as $t \rightarrow \infty$.

(iii) Let $y_{0}(\cdot)=0$ be a LAS solution of $(*)$. The domain of attraction of $y_{0}(\cdot)$ (or the domain of attraction of the set $\{0\}$ ) is the subset $A$ of $E$ consisting of all $a \in E$ such that the solution $y_{1}(\cdot)$ of $(*)$ starting at $a$ exists for every $t \geq 0$ and $y_{1}(t) \rightarrow 0$ as $t \rightarrow \infty$. (Note that if $E$ is open and $y_{0}(\cdot)$ is LAS, then the domain of attraction is also open.)

(iv) Assume that $E$ is an open set containing 0 and $F(0)=0$. Let $V$ be a function satisfying the following conditions:

(a) $V$ is defined in a neighbourhood $U$ of 0 ,

(b) $V$ is of class $\mathcal{C}^{1}$ in $U$,

(c) $V \geq 0$ and $V(y)>0$ if $\|y\|>0$,

(d) the trajectory derivative of $V$ at a point $y$ (i.e. $\dot{V}(y):=\frac{d}{d t} V[y(t)]=$ $\operatorname{grad} V(y) \circ F(y))$ is negative if $\|y\|>0$.

We call $V$ a Lyapunov function of the equation $(*)$.

Note that if all eigenvalues of $F^{\prime}(0)$ have negative real parts, then there exists a Lyapunov function of $(*)$.

Now we recall the following classical Lyapunov Theorem.

THEOREM 2.2. Let $E$ be an open set containing 0 , let $F$ be a $\mathcal{C}^{1}$ map of $\mathbb{R}^{n}$ with $F(0)=0$ and let $V$ be a Lyapunov function of the equation (*) defined in an open neighbourhood of 0 . Then $y(\cdot)=0$ is a LAS solution of $(*)$.

Let $E^{*}$ be a connected set. Let $G(y)=\left[g_{j k}(y): j, k=1, \ldots, n\right]$ be a real, symmetric, continuous and positive definite matrix on $E^{*}$. We associate with $G$ an elemēnt of arc length

$$
d s^{2}=d y^{T} G(y) d y,
$$


i.e. if $C: y=y(t), a \leq t \leq b$, is an arc of class $\mathcal{C}^{1}$ in $E^{*}$, then its length $L(C)$ is given by the formula

$$
L(C)=\int_{a}^{b}\left[\dot{y}(t)^{T} G(y(t)) \dot{y}(t)\right]^{1 / 2} d t
$$

$L(C)$ is independent of a chosen $\mathcal{C}^{1}$ parametrization of the arc $C$ (cf. [H]).

Take any $y_{1}, y_{2} \in E^{*}$ and define a metric associated with $G$ by the formula

$$
\begin{aligned}
r\left(y_{1}, y_{2}\right):=\inf \{L(C) \mid C:[a, b] & \rightarrow E^{*}, \\
y(a) & \left.=y_{1}, y(b)=y_{2}, C \text { is of class } \mathcal{C}^{1}\right\} .
\end{aligned}
$$

Now consider the equation $(*)$ and the "possible" Lyapunov function

$$
V(y):=F(y)^{T} G(y) F(y), \quad y \in E^{*} .
$$

Note that

$$
\dot{V}(y)=2 F(y)^{T} B(y) F(y)
$$

where

$$
B(y):=G(y) F^{\prime}(y)+\frac{1}{2} \sum_{j=1}^{n} F_{j} \frac{\partial G}{\partial y_{j}} .
$$

We recall the following correct version of Theorem 14.2 in [H, Chap. 14] (cf. also [HO, Theorem 2.4]).

THEOREM 2.3. Let $F=\left(F_{1}, \ldots, F_{n}\right)$ be a mapping of class $\mathcal{C}^{1}$. defined on an open connected subset $E^{*}$ of $\mathbb{R}^{n}$, with $F(y) \neq 0$ for every $y \in E^{*}$.

(i) Let a symmetric matrix $G(y)$ be of class $\mathcal{C}^{1}$ on $E^{*}$ and positive definite for $y \in E^{*}$, and let $B$ and $r$ be defined as above.

(ii) Assume that the following "Borg type" condition is satisfied:

$$
x^{T} B(y) x \leq 0 \quad \text { if } \quad F(y)^{T} G(y) x=0 .
$$

(iii) Further, let $y_{0}(\cdot)$ be a solution of $(*)$ defined on the right maximal interval of existence $0 \leq t<\omega \leq \infty$ with the property that there exists $\alpha>0$ such that

$$
r\left(y_{0}(t), \partial E^{*} \cup\{\infty\}\right)>\alpha>0 \quad \text { for every } t \in[0, \omega) ;
$$

i.e. for any $t \in[0, \omega)$ and for any half-open $\mathcal{C}^{1}$ arc $C: x=\phi(t), \phi(0)=$ $y_{0}(t), t \in[0,1)$, if $L(C) \leq \alpha$, then $\phi(1):=\lim _{t \rightarrow 1} \phi(t)$ exists and $\phi(t) \in E^{*}$ for $t \in[0,1]$.

Then there exist positive constants $\delta$ and $k$ such that for any solution $y(\cdot)$ of $(*)$ satisfying $r\left[y_{0}(0), y(0)\right]<\delta$ there exists an increasing, positive function $s(\cdot):[0, \omega) \rightarrow \mathbb{R}$ such that $s(0)=0,[0, s(\omega))$ is the right maximal 
interval of existence of $y(\cdot)$ and

$$
r\left[y(s(t)), y_{0}(t)\right] \leq k r\left[y(0), y_{0}(0)\right] \quad \text { for } 0 \leq t<\omega .
$$

3. Remarks on polynomial mappings. Let $F=\left(F_{1}, \ldots, F_{n}\right)$ be a polynomial map $\mathbb{R}^{n} \rightarrow \mathbb{R}^{n}$, and let Jac $F$ denote the jacobian of $F$, i.e. $\operatorname{Jac} F(x)=\operatorname{det} F^{\prime}(x)$ for $x \in \mathbb{R}^{n}$. We begin with the following lemma.

LEMMA 3.1. Let $F: \mathbb{R}^{n} \rightarrow \mathbb{R}^{n}$ be a polynomial map such that $\operatorname{det} F^{\prime}(y) \neq$ 0 for every $y \in \mathbb{R}^{n}$. Then for every $b \in \mathbb{R}^{n}$ the equation $F(x)=b$ has only isolated solutions and

$$
\#\left\{x \in \mathbb{R}^{n}: F(x)=b\right\} \leq \operatorname{deg} F_{1} \cdot \ldots \cdot \operatorname{deg} F_{n} .
$$

Moreover, $\left\{y \in \mathbb{R}^{n}: \# F^{-1}(y)=\max \left\{\# F^{-1}(b): b \in \mathbb{R}^{n}\right\}\right\}$ is a nonempty open subset of $\mathbb{R}^{n}$.

P r o of. Since Jac $F \neq 0$ everywhere in $\mathbb{R}^{n}$ the equation $F(x)=b$ has only isolated roots in $\mathbb{R}^{n}$. From now on we treat $F$ as a polynomial mapping of $\mathbb{C}^{n}$.

(i) If $F^{-1}(b)=\left\{a^{1}, \ldots, a^{p}: a^{j} \in \mathbb{C}^{n}, j=1, \ldots, p\right\}$, then the proof is given in [ $\mathrm{E}$, Chap. 7.13].

(ii) First we recall an important theorem about polynomial mappings (cf. [Md]):

If $F$ has a nontrivial jacobian and $d(F)=\left[\mathbb{C}\left(X_{1}, \ldots, X_{n}\right): \mathbb{C}\left(F_{1}, \ldots, F_{n}\right)\right]$, i.e. if $d(F)$ is the so-called geometric (or generic) degree of $F$, then

$$
d(F)=\max \left\{\# F^{-1}(y): y \in \mathbb{C}^{n}, \# F^{-1}(y)<\infty\right\}
$$

and the exceptional set $E:=\left\{y \in \mathbb{C}^{n}: \# F^{-1}(y) \neq d(F)\right\}$ is an algebraic proper subset of $\mathbb{C}^{n}$.

Assume that $\# F^{-1}(b)=\infty$ and let $a^{1}, \ldots, a^{p}$ be the isolated points of $F^{-1}(b)$. Choose closed balls $U_{j}=\bar{B}\left(a^{j}, R\right)$ such that

$$
U_{j} \cap F^{-1}(b)=\left\{a^{j}\right\}, \quad j=1, \ldots, p .
$$

Put $r:=\inf \left\{\|F(x)-b\|:\left\|x-a_{j}\right\|=R, j=1, \ldots, p\right\}>0$ and set $B=B(b, r)$. Choose $c \in B \backslash E$, fix $j$ and define holomorphic maps

$$
G:=b-c, \quad H:=F-b
$$

in some neighbourhood of $U_{j}$. Evidently

$$
\|G(x)\|<r \leq\|H(x)\| \text { when }\left\|x-a^{j}\right\|=R .
$$

By (1)-(3) we can apply the Rouché Theorem (cf. [E, Chap. 5]) to deduce that the holomorphic map $G+H=F-c$ has at least one zero in $U_{j}$, i.e.

$$
\#\left\{F^{-1}(c) \cap U_{j}\right\} \geq 1, \quad j=1, \ldots, p .
$$


From (4) we derive that

$$
p \leq \# F^{-1}(c)=d(F) \leq \operatorname{deg} F_{1} \cdot \ldots \cdot \operatorname{deg} F_{n} .
$$

Since $\#\left\{a \in \mathbb{R}^{n}: F(a)=b\right\} \leq \#\left\{c \in \mathbb{C}^{n}: F(c)=b\right\}$, we obtain the inequality stated in Lemma 3.1 .

(iii) Define $q:=\max \left\{\# F^{-1}(b): b \in \mathbb{R}^{n}\right\}$. Note that if a polynomial map $F$ of $\mathbb{R}^{n}$ has a nonvanishing jacobian, then by the local inverse function theorem $\left\{y \in \mathbb{R}^{n}: \# F^{-1}(y)=q\right\}$ is a nonempty open subset of $\mathbb{R}^{n}$.

Re mark 3.2. From the Lefschetz Principle we derive that the estimate given in Lemma 3.1 remains true for any field of characteristic 0 (cf. [E], where the existence of some estimate for any field of characteristic 0 is proved, and [BCR, Th. 11.5.2], where an estimate for the number of connected components of a real algebraic set is given).

It is worth remembering that injectivity of a polynomial map implies its bijectivity (cf. [BR, KR]).

4. Global stability of polynomial differential equations. We begin with a proposition which is a consequence of [HO, Theorems 2.2 and 2.4] or [H, Theorem 14.2 and Corollary 14.1].

Proposition 4.1. Assume that:

$1^{\circ} F=\left(F_{1}, \ldots, F_{n}\right)$ is a $\mathcal{C}^{1}$ map of $\mathbb{R}^{n}, F(0)=0$.

$2^{\circ}$ If $F(b)=0$, then $y(\cdot)=b$ is a LAS solution of the equation

$$
\dot{y}=F(y) \text {. }
$$

$3^{\circ} \exists p \in \mathcal{C}^{1}\left(\mathbb{R}^{n} \backslash F^{-1}(0),(0, \infty)\right)$ such that $\dot{p}(y)+p(y) x^{T} \circ F^{\prime}(y) \circ x \leq 0$ whenever $x^{T} \circ F(y)=0,\|x\|=1\left(\right.$ here $\left.\dot{p}(y):=p^{\prime}(y)^{T} \circ F(y)\right)$.

$4^{\circ} \exists R>0, \exists d>0$ such that $\|F(y)\|>d$ if $\|x\|>R$.

Then $y(\cdot)=0$ is a GAS solution of $(*)$. In particular, $F^{-1}(0)=\{0\}$.

Proof. (i) Evidently $\# F^{-1}(0)<\infty$, so $F^{-1}(0):=\left\{b_{1}=0, b_{2}, \ldots, b_{q}\right\}$. By $2^{\circ}$ the domain of attraction $D_{j}$ of the solution $y(\cdot)=b_{j}$ is nonempty for $j=1, \ldots, q$. Evidently $D_{i} \cap D_{j}=\emptyset$ for $i \neq j$. Put $E^{*}:=\mathbb{R}^{n} \backslash F^{-1}(0)$.

(ii) Define

$$
G(y):=p^{2}(y) I, \quad y \in E^{*} .
$$

Evidently $G(y)$ is a symmetric, positive definite matrix of class $\mathcal{C}^{1}$ on $E^{*}$ and, by $3^{\circ}, x^{T} \circ B(y) \circ x \leq 0$ whenever $F(y)^{T} \circ x=0,\|x\|=1$. Thus assumptions (i) and (ii) of Theorem 2.3 are satisfied.

(iii) If $q>1$, then $E^{*} \backslash D_{1} \neq \emptyset$. This means that there exists $y_{0} \in E^{*} \cap$ $\partial D_{1}$. Let $y_{0}(t)$ denote the solution of $(*)$ satisfying $y_{0}(0)=y_{0}$ and defined on $[0, \omega)$. Since $D_{1}$ is a domain of attraction, therefore, by the classical theorem 
on continuous dependence on initial values, $y_{0}(t) \in \partial D_{1}$ for $t \in[0, \omega)$. Now $4^{\circ}$ shows that

$\exists \alpha>0$ such that $\dot{r}\left(y_{0}(t), \partial E^{*} \cup\{\infty\}\right)>\alpha \quad$ for $0 \leq t<\omega$,

which means that assumption (iii) of Theorem 2.3 is satisfied.

(iv) By Theorem 2.3 there exist positive numbers $\delta$ and $k$ such that if $y(0) \in D_{1}$ and $r\left[y_{0}(0), y(0)\right]<\delta$ and $y(\cdot)$ is the solution of $(*)$ with initial value $y(0)$, then

$$
r\left[y(s(t)), y_{0}(t)\right] \leq k r\left[y(0), y_{0}(0)\right] \quad \text { for } 0 \leq t<\omega
$$

for a suitable increasing, positive function $s(\cdot):[0, \omega) \rightarrow(0, \infty)$ such that $s(0)=0$ and $[0, s(\omega))$ is the right maximal interval of existence of $y(\cdot)$. Since $y(t) \rightarrow 0$ as $t \rightarrow \infty$ we get a contradiction, hence $q=1$ and $y_{0}(\cdot)=0$ is a GAS solution in the whole $\mathbb{R}^{n}$.

Note that Proposition 4.1 remains true if instead of $4^{\circ}$ we assume that

$$
\forall r>0 \quad \int_{r}^{\infty} \varrho(s) d s=\infty, \quad \text { where } \varrho(s):=\min \{p(y):\|y\|=s\},
$$

but the proof is a little more complicated.

A typical candidate for $p$ is $p(y)=\|F(y)\|^{2 c}$, where $c$ is a fixed nonnegative number. Then $p(y)$ is positive of class $\mathcal{C}^{1}$, and $p$ satisfies $4^{\circ}$ if

$$
\begin{aligned}
& \dot{p}(y)+p(y) x^{T} \circ F^{\prime}(y) \circ x \\
& \quad=c\|F(y)\|^{2 c-2} F(y)^{T} \circ F^{\prime}(y) \circ F(y)+\|F(y)\|^{2 c} x^{T} \circ F^{\prime}(y) \circ x \leq 0 .
\end{aligned}
$$

This yields

Corollary 4.2. Proposition 4.1 remains true if $3^{\circ}$ is replaced by

(C) $\exists c \geq 0 \quad c F(y)^{T} \circ F^{\prime}(y) \circ F(y)+\|F(y)\|^{2} x^{T} \circ F^{\prime}(y) \circ x \leq 0$ whenever $x^{T} \circ F(y)=0,\|x\|=1$.

From Proposition 4.1, Theorem 2.2 and Corollary 4.2 we obtain at once the following.

Remark 4.3. Let $F=\left(F_{1}, \ldots, F_{n}\right)$ be a polynomial map of $\mathbb{R}^{n}$ with $F(0)=0$ such that

$$
\begin{gathered}
\operatorname{Jac} F(y) \neq 0 \quad \text { for every } y \in \mathbb{R}^{n}, \\
x^{T} \circ F^{\prime}(b) \circ x<0 \quad \text { if }\|x\|=1, F(b)=0, \\
\exists c \geq 0 \quad c[F(y)]^{T} \circ F^{\prime}(y) \circ F(y)+\|F(y)\|^{2} x^{T} \circ F^{\prime}(y) \circ x \leq 0 \\
\quad \text { whenever } x^{T} \circ F(y)=0,\|x\|=1 .
\end{gathered}
$$

Then $y(\cdot)=0$ is a GAS solution of $(*)$. In particular, $F^{-1}(0)=\{0\}$.

At this moment we want to show a connection between Borg type condition (C) and the eigenvalues of the matrix $H(y)$ which is the symmetric 
part of $F^{\prime}(y)$, i.e. $H(y)=\frac{1}{2}\left[F^{\prime}(y)+F^{\prime}(y)^{T}\right]$. Let $\lambda_{1}(y), \ldots, \lambda_{n}(y)$ be those eigenvalues. Define

$$
\alpha(y):=\max \left\{\left(\lambda_{j}(y)+\lambda_{k}(y)\right): j \neq k, j, k=1, \ldots, n\right\}, \quad y \in \mathbb{R}^{n} .
$$

Re mark 4.4. Inequality (2.5) in [HO] implies

$$
\begin{array}{r}
F(y)^{T} \circ F^{\prime}(y) \circ F(y)+\|F(y)\|^{2} x^{T} \circ F^{\prime}(y) \circ x \leq \alpha(y)\|F(y)\|^{2} \\
\text { whenever } x^{T} \circ F(y)=0,\|x\|=1 .
\end{array}
$$

Now we formulate and prove the main theorem of the paper.

THEOREM 4.5 (Main Theorem). Let $F=\left(F_{1}, \ldots, F_{n}\right)$ be a polynomial map of $\mathbb{R}^{n}, F(0)=0$. Let $H(y), \lambda_{1}(y), \ldots, \lambda_{n}(y)$ and $\alpha(y)$ be as defined above. Assume that

(a) $\mathrm{Jac} F(y) \neq 0$ for every $y \in \mathbb{R}^{n}$,

(b) all eigenvalues of $F^{\prime}(b)$ have negative real parts if $F(b)=0$,

(c) $\alpha(y) \leq 0$ for every $y \in \mathbb{R}^{n}$.

Then $y(\cdot)=0$ is a GAS solution of $(*), y \in \mathbb{R}^{n}$, and the mapping $F$ is bijective.

Proof. Case I. We assume additionally $\# F^{-1}(0)=\max \left\{\# F^{-1}(w)\right.$ : $w \in \mathbb{R}^{n}$ \}. Now it is sufficient to prove that $y_{0}(\cdot)=0$ is a GAS solution of (*). By (b) and Theorem 2.2, $y(\cdot)=b$ is a LAS solution of $(*)$ whenever $F(b)=0$.

We check that the function

$$
p(y):=\|F(y)\|^{2}, \quad y \in \mathbb{R}^{n} \backslash F^{-1}(0),
$$

satisfies the assumptions of Corollary 4.2. By Remark 4.4,

$$
\begin{aligned}
\dot{p}(y)+p(y) x^{T} \circ F^{\prime}(y) \circ x & =F(y)^{T} \circ F^{\prime}(y) \circ F(y)+\|F(y)\|^{2} x^{T} \circ F^{\prime}(y) \circ x \\
& \leq \alpha(y)\|F(y)\|^{2} \leq 0
\end{aligned}
$$

whenever $x^{T} \circ F(y)=0,\|x\|=1$. Therefore, assumption (C) of Corollary 4.2 is satisfied.

Since $\# F^{-1}(0)=\max \left\{\# F^{-1}(w): w \in \mathbb{R}^{n}\right\}$, there exists $d>0$ such that $A:=\left\{y \in \mathbb{R}^{n}:\|F(y)\| \leq d\right\}$ is compact. Hence,

$$
\exists R>0 \text { such that }\|F(y)\|>d \text { if }\|x\|>R,
$$

i.e. $4^{\circ}$ is also satisfied. Thus, by Corollary $4.2, y_{0}(\cdot)=0$ is a GAS solution of $(*)$ and $F$ is bijective.

C a s e II. If $\# F^{-1}(0)<\max \left\{\# F^{-1}(w): w \in \mathbb{R}^{n}\right\}$, then, by Lemma 3.1, we can choose $b \in \mathbb{R}^{n}$ such that $\# F^{-1}(b)=\max \left\{\# F^{-1}(w): w \in \mathbb{R}^{n}\right\}$. Put

$$
G(y):=F(b+y)-F(b), \quad y \in \mathbb{R}^{n} .
$$


Obviously $G$ satisfies all assumptions which $F$ satisfied in Case I. Hence $G$ is bijective, so is $F$, and $F$ has to satisfy the assumptions of Case $I$.

Now we prove that Theorem 4.5 generalizes Meisters and Olech's result (cf. $[\mathrm{MO}]$ ).

TheOREM 4.6. Let $F=\left(F_{1}, F_{2}\right)$ be a polynomial mapping of $\mathbb{R}^{2}$. If $F(0)=0, \operatorname{Jac} F(y)>0$ and

$$
\operatorname{Tr} F^{\prime}(y):=\frac{\partial F_{1}}{\partial y_{1}}(y)+\frac{\partial F_{2}}{\partial y_{2}}(y)<0 \quad \text { for every } y \in \mathbb{R}^{2},
$$

then $y(\cdot)=0$ is a GAS solution of the autonomous system

$$
\dot{y}_{1}=F_{1}\left(y_{1}, y_{2}\right), \quad \dot{y}_{2}=F_{2}\left(y_{1}, y_{2}\right), \quad\left(y_{1}, y_{2}\right) \in \mathbb{R}^{2} \text {. }
$$

In particular, $F^{-1}(0)=\{0\}$.

Proof. We show that the assumptions of Theorem 4.6 imply those of Theorem 4.5 for $n=2$.

Since $\operatorname{Jac} F(y)>0$ and $\operatorname{Tr} F^{\prime}(y)<0$, all eigenvalues of $F^{\prime}(y)$ have negative real parts. If $H(y):=\frac{1}{2}\left[F^{\prime}(y)+F^{\prime}(y)^{T}\right], y \in \mathbb{R}^{n}$, and $\lambda_{1}(y), \lambda_{2}(y)$ are the eigenvalues of $H(y)$, then

$$
\alpha(y)=\lambda_{1}(y)+\lambda_{2}(y)=\operatorname{Tr} H^{\prime}(y)=\operatorname{Tr} F^{\prime}(y)<0 .
$$

Thus all assumptions of Theorem 4.5 are satisfied.

It is known that if a complex polynomial map of $\mathbb{C}^{2}$ has nontrivial constant jacobian and symmetric jacobian matrix, then the map is bijective (cf. [D]). For the sake of comparison we present a real counterpart of this fact.

Remark 4.7. If $F$ is a polynomial mapping of $\mathbb{R}^{2}, \operatorname{Jac} F(y)>0$ and $F^{\prime}(y)$ is symmetric for any $y \in \mathbb{R}^{2}$, then $F$ is bijective.

One can check this by applying Theorem 4.6. The result is also true for any $\mathcal{C}^{1}$ map and it is a consequence of Corollary 1 in [MO1].

Note that assumption (C) of Corollary 4.2 in fact concerns the symmetric part $H(y)$ of $F^{\prime}(y)$ because

$$
\begin{aligned}
B(x, y) & :=c F(y)^{T} \circ F^{\prime}(y) \circ F(y)+\|F(y)\|^{2} x^{T} \circ F^{\prime}(y) \circ x \\
& =c F(y)^{T} \circ H^{\prime}(y) \circ F(y)+\|F(y)\|^{2} x^{T} \circ H^{\prime}(y) \circ x
\end{aligned}
$$

thus, it can be very restrictive even in the case of a linear system of differential equations. The following simple example, presented to us by A. van den Essen, shows this fact.

EXAMPLE 4.8. Define the linear mapping $F$ of $\mathbb{R}^{3}$ by

$$
F\left(y_{1}, y_{2}, y_{3}\right)=\left(-y_{1}+21 y_{3},-y_{2}+12 y_{3},-y_{3}\right) \text {. }
$$


Then the zero solution of the equation $\dot{y}=F(y)$ is globally asymptotically stable, but assumption (C) of Corollary 4.2 is not satisfied because if

$$
x=\frac{1}{\sqrt{2}}(1,1,0) \quad \text { and } \quad y=\frac{-1}{\sqrt{3}}(22,11,1) \text {, }
$$

then

$$
F(y)=\frac{-1}{\sqrt{3}}(-1,1,-1), \quad x^{T} \circ F(y)=0, \quad B(x, y)=2 c-1,
$$

while if

$$
x=\frac{1}{\sqrt{3}}(1,-1,1) \quad \text { and } \quad y=\frac{-1}{\sqrt{2}}(1,1,0),
$$

then

$$
F(y)=\frac{-1}{\sqrt{2}}(-1,-1,0), \quad x^{T} \circ F(y)=0, \quad B(x, y)=2-c .
$$

Acknowledgement. The authors wish to thank K. Holly for helpful discussions and the referee for valuable suggestions and remarks.

\section{References}

[B] N. E. Barabanov, On Kalman's problem, Sibirsk. Mat. Zh. 29 (3) (1988), 2-11 (in Russian).

[BR] A. Białynicki-Birula and M. Rosenlicht, Injective morphisms of real algebraic varieties, Proc. Amer. Math. Soc. 13 (1962), 200-203.

[BCR] J. Bochnak, M. Coste et M.-F. Roy, Géométrie Algébrique Réelle, Springer, Berlin 1987.

[D] F. Dillen, Polynomials with constant Hessian determinant, J. Pure Appl. Algebra 71 (1991), 13-18.

[E] A. van den Essen, A note on Meisters and Olech's proof of the global asymptotic stability Jacobian conjecture, Pacific J. Math. 151 (1991), 351-356.

[H] P. Hartman, Ordinary Differential Equations, Wiley, New York 1964.

[HO] P. Hartman and C. Olech, On global asymptotic stability of solutions of differential equations, Trans. Amer. Math. Soc. 104 (1962), 154-178.

[KR] K. Kurdyka and K. Rusek, Surjectivity of certain injective semialgebraic transformations of $\mathbb{R}^{n}$, Math. Z. 200 (1988), 141-148.

[E] S. Lojasiewicz, Introduction to Complex Analytic Geometry, Birkhäuser, Basel 1991.

[MY] L. Markus and H. Yamabe, Global stability criteria for differential systems, Osaka Math. J. 12 (1960), 305-317.

[MO] G. H. Meisters and C. Olech, Solution of the global asymptotic stability Jacobian conjecture for the polynomial case, in: Analyse Mathématique et Applications, Gauthier-Villars, Paris 1988, 373-381.

[MO1] -, -, A Jacobian condition for injectivity of differentiable plane maps, Ann. Polon. Math. 51 (1990), 249-254.

[Md] D. Mumford, Algebraic Geometry, I. Complex Projective Varieties, Springer, Berlin 1976. 
[O] C. Olech, On the global stability of an autonomous system on the plane, Contributions Differential Equations 1 (1963), 389-400.

[P] T. Parthasarathy, On Global Univalence, Lecture Notes in Math. 977, Springer, Berlin 1983.

INSTITUTE OF MATHEMATICS

JAGIELLONIAN UNIVERSITY

REYMONTA 4

30-059 KRAKÓW, POLAND

E-mail: UMDRUZKO@PLKRCY11.BITNET and UMTUTAJ@PLKRCY11.BITNET

Reçu par la Rédaction le 30.10.1991

Révisé le 15.6.1992 